\title{
Aspectos peculiares do traumatismo facial no idoso: Relato de caso
}

\author{
Peculiar aspects of facial trauma in the elderly: Case report \\ Aspectos peculiares del traumas em el anciano: Reporte de um caso
}

Recebido: 22/05/2021 | Revisado: 29/05/2021 | Aceito: 07/06/2021 | Publicado: 21/06/2021

Ingrid Aquino Moreira de Sousa ORCID: https://orcid.org/0000-0001-8377-1852 Universidade de Pernambuco, Brasil E-mail: ingrid.aquino@upe.br

Ramisse Moreira de Albuquerque ORCID: https://orcid.org/0000-0002-7647-4953 Universidade de Pernambuco, Brasil E-mail: ramisse.albuquerque @upe.br

Ingrid Patrícia de Moraes Lima ORCID: https://orcid.org/0000-0003-0740-3518 Universidade de Pernambuco, Brasil E-mail: ingrid.moraes@upe.br Sarah Freitas Araújo ORCID: https://orcid.org/0000-0002-4491-3210 Universidade de Pernambuco, Brasil E-mail: sarah.freitas@upe.br

Thais Aquino Moreira de Sousa ORCID: https://orcid.org/0000-0002-2974-5378 Universidade de Pernambuco, Brasil E-mail: aquino95thais@gmail.com

Emerson Filipe de Carvalho Nogueira ORCID: https://orcid.org/0000-0002-4560-7733 Universidade de Pernambuco, Brasil E-mail: emerson_filipe@ hotmail.com

\section{Resumo}

Este caso clínico relata tratamento cirúrgico em paciente idosa desdentada bimaxilar após trauma em terço inferior de face. A paciente compareceu a emergência do Hospital de Fraturas, Recife - PE com queixa de dor em face após queda da própria altura. Ao exame radiográfico, constatou-se fratura mandibular bilateral. O planejamento do tratamento foi realizado levando em consideração alguns fatores importantes para o sucesso terapêutico, como uso de medicamentos, presença de doenças crônicas e alterações fisiológicas e anatômicas. Após o diagnóstico e correto planejamento, foi realizada a fixação dos cotos ósseos com 2 miniplaca do sistema 2.0. A paciente evoluiu bem, sem queixas, sem parestesia ou paralisia, e a radiografia pós-operatória demonstrou boa redução das fraturas. Dessa forma, conclui-se que o tratamento dos traumas em pacientes idosos pode ser através de métodos conservadores ou de técnicas mais complexas e invasivas, levando-se sempre em consideração o caso particular do paciente, visando suas necessidades e implicações específicas.

Palavras-chave: População idosa; Traumatismo; Fratura de ossos.

\begin{abstract}
This clinical case reports surgical treatment in an elderly toothless patient after trauma to the lower third of the face. The patient attended the emergency at the Hospital de Fraturas, Recife - PE, complaining of pain in the face after falling from her own height. Radiographic examination revealed bilateral mandibular fracture. Treatment planning was carried out taking into account some important factors for therapeutic success, such as the use of medications, the presence of chronic diseases and physiological and anatomical changes. After diagnosis and correct planning, bone stumps were fixed with two 2.0 system mini-plates. The patient evolved well, without complaints, without paresthesia or paralysis, and the postoperative radiography demonstrated a good reduction in fractures. Thus, it is concluded that the treatment of trauma in elderly patients can be through conservative methods or more complex and invasive techniques, always taking into account the patient's particular case, aiming at their specific needs and implications.
\end{abstract}

Keywords: Elderly population; Trauma; Bone fracture.

\section{Resumen}

Este caso clínico reporta el tratamiento quirúrgico en un paciente anciano bimaxilar desdentado tras traumatismo en el tercio inferior de la cara. La paciente acude a urgencias en el Hospital de Fraturas, Recife - PE, quejándose de 
dolor en el rostro tras caer de su propia altura. El examen radiográfico reveló fractura mandibular bilateral. La planificación del tratamiento se llevó a cabo teniendo en cuenta algunos factores importantes para el éxito terapéutico, como el uso de medicamentos, la presencia de enfermedades crónicas y cambios fisiológicos y anatómicos. Tras el diagnóstico y la correcta planificación, los muñones óseos se fijaron con 2 miniplacas del sistema 2.0. El paciente evolucionó bien, sin quejas, sin parestesias ni parálisis, y la radiografía postoperatoria demostró una buena reducción de fracturas. Así, se concluye que el tratamiento del trauma en el anciano puede ser a través de métodos conservadores o técnicas más complejas e invasivas, siempre teniendo en cuenta el caso particular del paciente, atendiendo a sus necesidades e implicaciones específicas.

Palabras clave: Población anciana; Trauma; Fractura ósea.

\section{Introdução}

A Organização Mundial de Saúde define idosos como um grupo de indivíduos com mais de 60 anos que vivem em países em desenvolvimento, enquanto nos países desenvolvidos, estende-se para 65 anos de idade (WHO, 2016).

Com a mudança na distribuição de idade da população mundial, os avanços da medicina e a expectativa de vida prolongada, a proporção de pessoas idosas está crescendo em todo o mundo. Estima-se que, em 2025, aumentos de até 300\% ocorram na população idosa em países em desenvolvimento e que em 2050, haverá dois bilhões de pessoas com mais de 60 anos, sendo $80 \%$ destas vivendo em países em desenvolvimento (Chrcanovic et al., 2010). Diante desse contexto, o crescente número de idosos acaba por refletir no perfil de pacientes atendidos na área de traumatologia bucomaxilofacia (de Sousa, 2019).

O trauma facial acomete mais comumente adultos jovens urbanos. Nos últimos anos, no entanto, esse estereótipo tem sido questionado, pois há uma incidência crescente deste tipo de trauma em idosos que se tornam mais expostos aos agentes agressores da vida moderna, tornando-os alvos de diversos tipos de traumatismos, incluindo os bucomaxilofaciais (Chrcanovic et al., 2010). Existem, porém, poucos artigos publicados que abordam o impacto do envelhecimento sobre o trauma facial em pacientes idosos e há deficiência de estudos avaliando incidência, causa, padrões de fratura e seu gerenciamento em pacientes geriátricos (Berg et al., 2014; Carvalho Filho et al., 2015).

O trauma é a sétima causa mais comum de morte em pessoas idosas, sendo responsável por $25 \%$ dos óbitos nessa faixa etária (Fonseca et al, 2015). O cuidado a esses pacientes representa um desafio para os profissionais de saúde e estudos apontam que o trauma nesses pacientes está associado a longas estadias hospitalares e custo elevado (Toivari et al, 2016; Velayutham et al., 2013). Vale ressaltar, também, que a alta mortalidade e morbidade são complicações associadas a infecções hospitalares adquiridas (Velayutham et al., 2013).

Diante do atual panorama da população idosa mundial, o presente estudo tem como objetivo avaliar os aspectos peculiares do traumatismo facial do idoso, a fim de orientar a conduta mais adequada que o cirurgião bucomaxilofacial deve adotar.

\section{Metodologia}

O presente artigo trata-se de um relato de caso clínico, pois descreve e analisa de forma detalhada caso que de trauma facial em idosa (Pereira et al, 2018). O estudo tem caráter qualitativo visto que se fundamenta nas interpretações por parte dos pesquisadores sobre o caso estudado (Ludke \& Andre, 2013). A paciente concordou com a participação na pesquisa e assinou um termo de consentimento livre e esclarecido.

\section{Relato de Caso}

Paciente leucoderma, sexo feminino, 76 anos de idade, hipertensa, compareceu a emergência do Hospital de Fraturas, Recife - PE, apresentando como queixa dor em face após queda da própria altura. Ao exame físico facial, apresentava 
equimose em região mandibular, hematoma em assoalho bucal, crepitação em corpo mandibular bilateral com parestesia do lado esquerdo.

Figura 1. Estado inicial da paciente extra e intra-bucal.

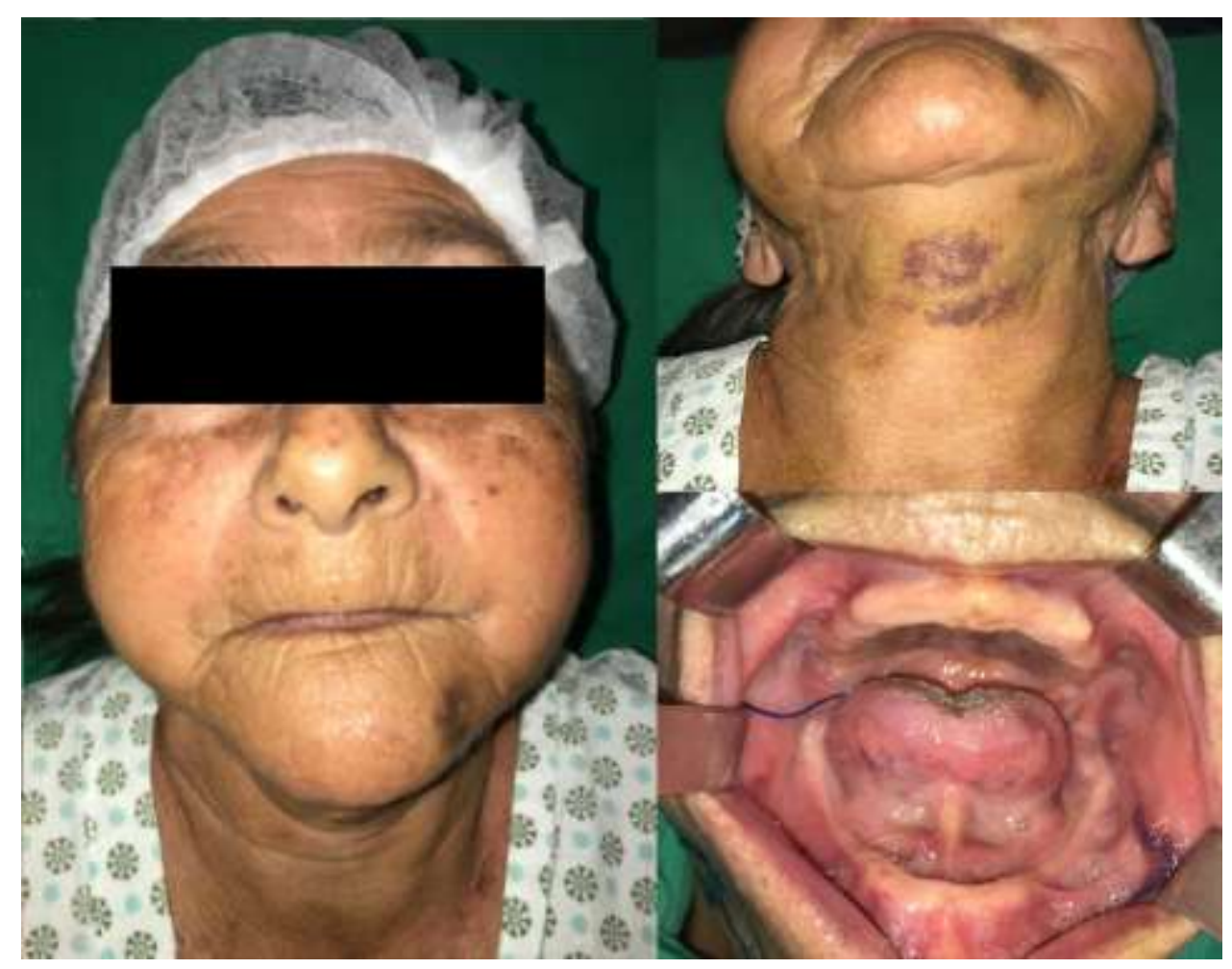

Fonte: Autores.

Foi solicitado radiografia PA de mandíbula, como também lateral oblíqua de mandíbula direita e esquerda, a qual pode-se evidenciar perda de continuidade óssea em corpo mandibular bilateral, confirmando assim o diagnóstico de fratura mandibular bilateral em paciente desdentada bimaxilar. Também foram solicitados exames pré-operatórios laboratoriais e parecer cardiológico.

Uma semana após, a paciente foi submetida a anestesia geral sob intubação nasotraqueal. Optou-se pelo acesso cirúrgico submandibular, seguido pelo divulsionamento por planos até localização da fratura. Realizou-se a curetagem do tecido de granulação e redução anatômica dos cotos fraturados, estabilização com 1 miniplaca do sistema 2.0 na zona de tensão e outra na zona de compressão. A mesma técnica foi realizada no lado contralateral e a ferida foi suturada por planos. 
Figura 2. A) Trauma no corpo da mandíbula do lado esquerdo; B) Trauma no corpo da mandíbula do lado direito; C) Estabilização da fratura no lado esquerdo em vista frontal; D) Estabilização da fratura no lado esquerdo em vista lateralizada; E) Estabilização da fratura no lado direito em vista frontal; F) Estabilização da fratura no lado direito em vista lateralizada.

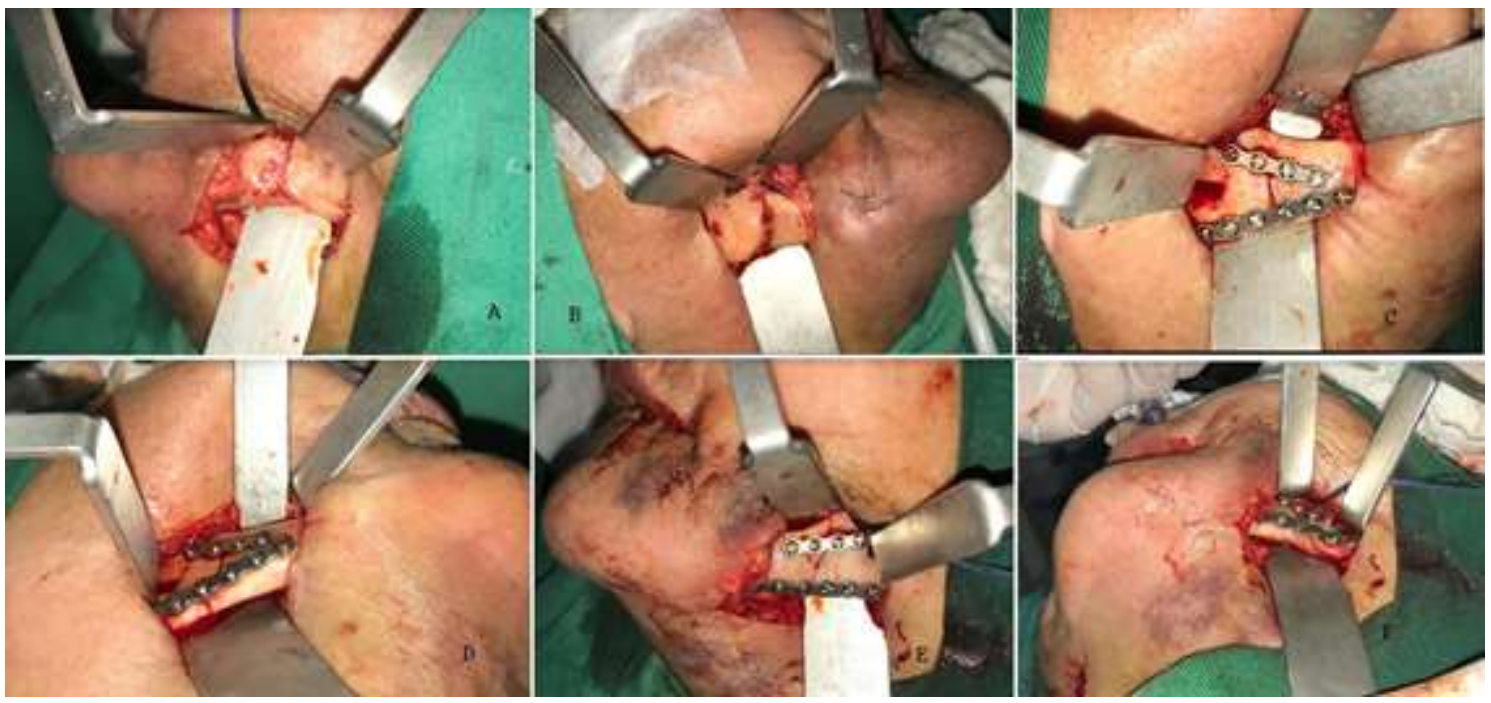

Fonte: Autores.

A paciente evoluiu bem, sem queixas, sem parestesia ou paralisia, e a radiografia pós-operatória demonstrou boa redução das fraturas.

Figura 3. A) Fratura do lado direito antes e após a estabilização. B) Fratura do lado esquerdo antes e após a estabilização.

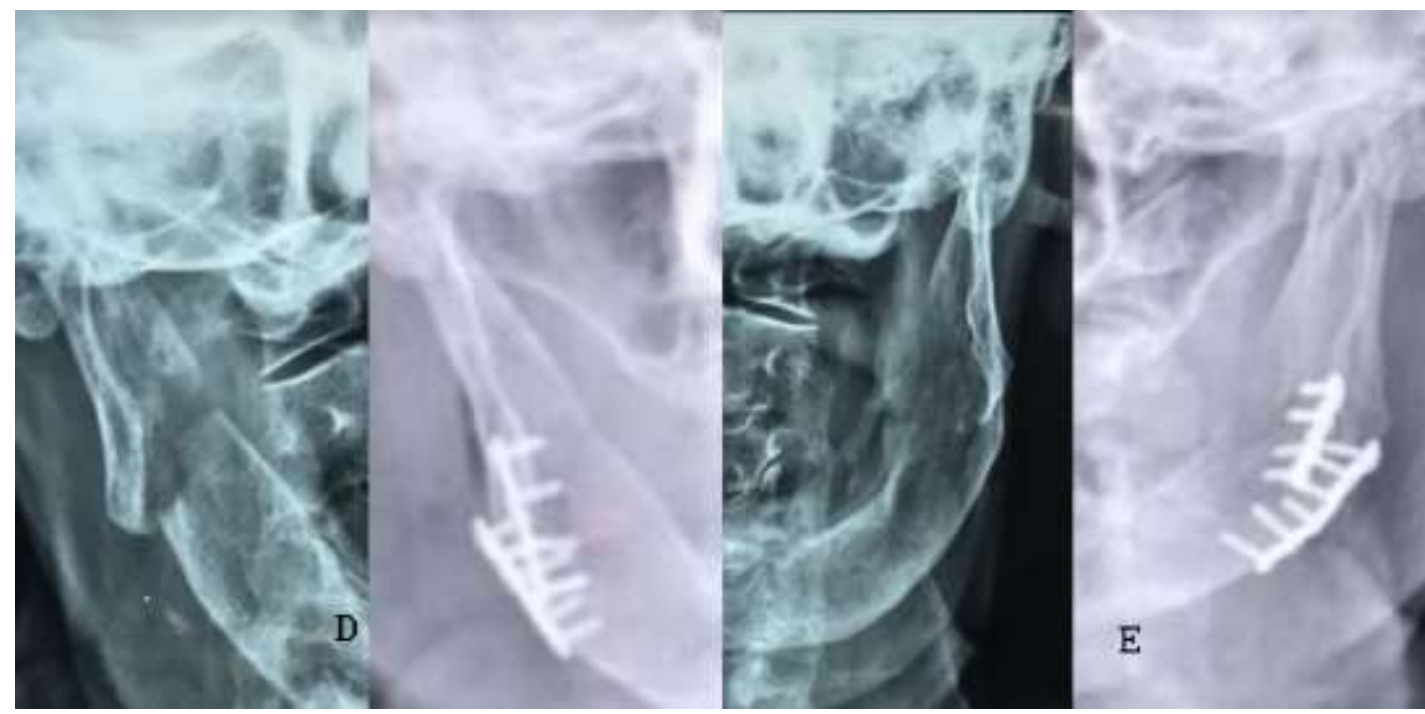

Fonte: Autores.

\section{Discussão}

A expectativa de vida na América é maior para as mulheres do que para os homens, o que corresponde ao aumento do número de mulheres idosas com trauma de face (Berg et al., 2014). Além disso, comparado com os homens, a perda de massa óssea em mulheres começa mais cedo (a quarta década em comparação com a quinta) e é mais drástico (2 a 3 por cento ao ano em comparação com 0,4 por cento para homens). Tal fato pode ser justificado pela osteoporose senil e pós-menopausa que podem ocorrer em mulheres idosas (Atisha et al., 2012). 
Uma compreensão completa da epidemiologia e padrões de trauma facial na população idosa é essencial para um manejo mais adequado desses pacientes e para desenvolver estratégias de tratamento mais eficazes e possivelmente prevenir lesões (Brucoli et al., 2020a).

Os principais fatores etiológicos do trauma são quedas da própria altura e acidentes de trânsito (Giacomin et al., 2017). Vale destacar que a violência física ganhou maior notoriedade social devido ao envelhecimento crescente da população que caracteriza a sociedade moderna (de Sousa et al, 2016).

O estilo de vida e hábitos relacionados à idade predispõe os idosos a acidentes domésticos e traumas de menor energia cinética (Giacomin et al., 2017). As quedas são frequentes nessa faixa etária, principalmente no banheiro (Hwang et al., 2013; Hanba et al., 2017). Problemas cardiovasculares e acidentes vasculares cerebrais, o decréscimo na massa óssea e força muscular, assim como características inerentes ao processo de envelhecimento (diminuição da propriocepção, alterações na resposta motora, tremores, diminuição da acuidade visual e auditiva) predispõem a um maior número de quedas e tropeços, como mostra os estudos de Berg et al e Arangio, et al, podendo chegar a 90\% dos casos (Giacomin et al., 2017; Arangio et al., 2012).

O trauma na região facial frequentemente resulta em injúrias ao tecido mole, aos dentes e aos ossos da face, incluindo mandíbula, maxila, zigomático, complexo naso-órbito-etmoidal e estruturas supraorbitárias· (Giacomin et al., 2017). Há uma diminuição da força do esqueleto facial dos idosos, pois o osso torna-se mais poroso, há expansão no volume da cavidade paranasal e, simultaneamente, reabsorção progressiva do osso alveolar bimaxilar, principalmente nas regiões edêntulas (Arangio et al., 2012).

Em virtude da dificuldade em amortecer a queda, o osso nasal e zigomático, ossos mais salientes do que outras estruturas da face, e a órbita, que cobre uma área de superfície relativamente maior, podem ser fraturados (Liu et al., 2017). Sabe-se que atrofia progressiva da mandíbula (especialmente em pacientes edêntulos) tem influência na incidência de fratura de côndilo e corpo mandibular (Brucoli et al., 2020a).

Os princípios do manejo das fraturas faciais na população idosa e em adultos são basicamente os mesmos, mas o transoperatório em geriátricos torna-se mais complexo devido às variações anatômicas e comorbidades médicas, o que ocasiona um consumo desproporcional de recursos de saúde (Brucoli et al., 2020b). Esses pacientes são mais suscetíveis a infecções nosocomiais, além de apresentarem maiores taxas de complicações e maior tempo de recuperação (Liu et al., 2017). É fundamental o planejamento para execução do tratamento, incluindo a avaliação do estado médico desses pacientes com lesão em face (Brucoli et al., 2020a).

As mudanças fisiológicas que ocorrem com a idade podem aumentar a incidência de complicações e diminuir a chance de sobrevida. O cirurgião bucomaxilofacial deve considerar as condições sistêmicas para tratar pacientes idosos e a assistência deve ser diferenciada (Giacomin et al., 2017). Pacientes geriátricos com fraturas faciais representam um desafio adicional, visto que seu manejo deve considerar atrofia óssea, número limitado de dentes residuais, capacidade reduzida para a reparação tecidual e aumento do risco de infecção (Liu et al., 2017; Nogami et al., 2015).

Esses pacientes comumente apresentam hipertensão, dislipidemias e diabetes mellitus, que contribuem para aumento da morbidade (Liu et al., 2017; Nogami et al., 2015). As doenças cardiovasculares e as alterações pulmonares e renais são comumente encontradas e podem alterar o tratamento ou limitá-lo à terapia pouco invasiva (Giacomin et al., 2017). É importante destacar que o tratamento de condições cardiovasculares é frequentemente associado ao uso de drogas antitrombóticas, que precisam ser levadas em consideração antes da cirurgia (Brucoli et al., 2020a).

Doença periodontal, presença de próteses e a ausência de dentes podem comprometer o bloqueio maxilomandibular. Além disso, deve-se considerar tempo de imobilização mais longos para a consolidação da fratura. É utilizado rotineiramente a fixação interna rígida com placas e parafusos para estabilização das fraturas faciais (Chrcanovic et al., 2010). 
A cirurgia é indicada na presença de déficits funcionais, deslocamento significativo da fratura, risco de sepse ou falta de consolidação óssea. Entretanto, deve-se considerar fatores como atrofia óssea, capacidade reduzida de reparo de tecidos e condições patológicas (Chrcanovic et al., 2010). Quando a equipe julga que uma intervenção cirúrgica complexa pode representar uma ameaça à vida ou que os riscos da cirurgia podem superar suas vantagens, a su spensão desse tratamento é uma alternativa prudente (Brucoli et al., 2020b).

São indicações ao tratamento cirúrgico nas fraturas de mandíbula: fraturas desfavoráveis, expostas e/ou com limitação dos movimentos mandibulares. Em pacientes geriátricos, a mudança na oclusão não é uma indicação completa para cirurgia porque, muitas vezes, são edêntulos ou parcialmente edêntulos. A cirurgia é indicada quando as alterações de oclusão determinam restrição funcional (Arangio et al., 2012).

A instabilidade inerente das fraturas de corpo de mandíbula atrófica torna imprevisível a manutenção de uma redução adequada e imobilização sem a estabilização da mesma. A realização de fixação interna rígida, portanto, deve ser realizada. É importante pontuar, também, que a posição do nervo alveolar inferior, pode dificultar a fixação interna (Fonseca et al, 2015).

\section{Considerações Finais}

O tratamento do trauma facial em idosos visa preservar e restaurar a função, melhorando a qualidade de vida, logo, a escolha do método de tratamento para as fraturas faciais deve considerar os aspectos inerentes ao envelhecimento e a presença de doenças sistêmicas, que podem aumentar a morbidade e complicações. O crescimento da população idosa e a melhor qualidade de vida desse grupo estão associados à exposição a agentes agressores como, por exemplo, casos de idosos que sofrem traumatismo facial, assim, recomenda-se mais estudos acerca desse tema, considerando implicações específicas dessa faixa etária.

\section{Referências}

Arangio, P., Leonardi, A., Torre, U., Bianca, C., \& Cascone, P. (2012). Management of facial trauma in patients older than 75 years. Journal of Craniofacial Surgery, 23(6), 1690-1692.

Atisha, D., Allori, A., Nolen, D., Ruane, E., Christie, B., Sharma, K., ... \& Erdmann, D. (2012). Facial Fractures in the Aging Population. Plastic and Reconstructive Surgery, 130(5S-1), 6.

Berg, B. I., Juergens, P., Soerensen, Y., Savic, M., Zeilhofer, H. F., \& Schwenzer-Zimmerer, K. (2014). Traumatology of the facial skeleton in octogenarian patients: a retrospective analysis of 96 cases. Journal of Cranio-Maxillofacial Surgery, 42(6), 870-873.

Brucoli, M., Boffano, P., Romeo, I., Corio, C., Benech, A., Ruslin, M., ... \& Dediol, E. (2020a). Epidemiology of maxillofacial trauma in the elderly: A European multicenter study. Journal of stomatology, oral and maxillofacial surgery, 121(4), 330-338.

Brucoli, M., Boffano, P., Romeo, I., Corio, C., Benech, A., Ruslin, M., ... \& Konstantinovic, V. S. (2020b). Management of maxillofacial trauma in the elderly: A European multicenter study. Dental traumatology, 36(3), 241-246.

Carvalho Filho, M. A. M., de Lima Saintrain, M. V., Dos Anjos, R. E. D. S., Pinheiro, S. S., Cardoso, L. D. C. P., Moizan, J. A. H., \& de Aguiar, A. S. W. (2015). Prevalence of oral and maxillofacial trauma in elders admitted to a reference hospital in northeastern Brazil. PLoS one, 10(8), e0135813.

Chrcanovic, B. R., Souza, L. N., Freire-Maia, B., \& Abreu, M. H. N. G. (2010). Facial fractures in the elderly: a retrospective study in a hospital in Belo Horizonte, Brazil. Journal of Trauma and Acute Care Surgery, 69(6), E73-E78.

Fonseca, R. J., Walker, R. J., Barber, H. D., Powers, M. P., \& Frost, D. E. (2015). Trauma bucomaxilofacial. Elsevier Brasil.

Giacomin, M., Conto, F. D., Siqueira, S. P., Signori, P. H., Eidt, J. M. S., \& Sawazaki, R. (2017). Trauma facial em idosos: uma análise retrospectiva de 10 anos. Revista Brasileira de Geriatria e Gerontologia, 20(5), 618-623.

Hanba, C., Gupta, A., Svider, P. F., Folbe, A. J., Eloy, J. A., Zuliani, G. F., \& Carron, M. A. Forgetful but not forgotten: Bathroom-related craniofacial trauma among the elderly. The Laryngoscope. 2017; 127(4), 820-827.

Hwang, K., Huan, F., \& Hwang, P. J. (2013). Comparison of facial trauma in late middle age (55-64 years) and old age (older than 65 years). Journal of Craniofacial Surgery, 24(3), 909-913. 
Research, Society and Development, v. 10, n. 7, e27810716385, 2021

(CC BY 4.0) | ISSN 2525-3409 | DOI: http://dx.doi.org/10.33448/rsd-v10i7.16385

Liu, F. C., Halsey, J. N., Oleck, N. N., Hoppe, I. C., Lee, E. S., \& Granick, M. S. (2017). Facial Fractures as a Result of Falls in the Elderly: Concomitant Injuries and Management Strategies. Plastic and Reconstructive Surgery Global Open, 5 (9 Suppl).

Ludke, M. \& Andre, M. E. D. A. (2013). Pesquisas em educação: uma abordagem qualitativa. São Paulo: E.P.U. 5.5) Yin, R.K. (2015)

Nogami, S., Yamauchi, K., Yamashita, T., Kataoka, Y., Hirayama, B., Tanaka, K., \& Takahashi, T. (2015). Elderly patients with maxillofacial trauma: study of mandibular condyle fractures. Dental traumatology, 31(1), 73-76.

Pereira A. S. et al. (2018). Metodologia da pesquisa científica. [free e-book]. Santa Maria/RS. Ed. UAB/NTE/UFSM.

Sousa Leite, P., Gonçalves, D. V. L. G., Magalhães, B. F., Cruz, E. F., Cruz, A. F., Balduino, C. H. C., ... \& de Alencar, T. M. (2019). Fatores Epidemiológicos do Trauma em Pacientes Idosos Atendidos em Serviços de Emergência/Epidemiological Factors of Trauma in Elderly Patients Cared for in Emergency Services. ID on line REVISTA DE PSICOLOGIA, 13(48), 156-167.

Sousa, R. I. M., de Macedo Bernardino, Í., de Castro, R. D., Cavalcanti, A. L., Bento, P. M., \& d'Ávila, S. (2016). Maxillofacial trauma resulting from physical violence against older adults: A 4-year study in a Brazilian forensic service. Pesquisa Brasileira em Odontopediatria e Clínica Integrada, 16(1).

Toivari, M., Suominen, A. L., Lindqvist, C., \& Thorén, H. (2016). Among patients with facial fractures, geriatric patients have an increased risk for associated injuries. Journal of Oral and Maxillofacial Surgery, 74(7), 1403-1409.

World health organization (Geneva). World health statistics 2016: monitoring health for the SDGs, sustainable development goals. World Health Organization, Geneva, 2015.

Velayutham, L., Sivanandarajasingam, A., O’Meara, C., \& Hyam, D. (2013). Elderly patients with maxillofacial trauma: the effect of an ageing population on a maxillofacial unit's workload. British journal of oral and maxillofacial surgery, 51(2), 128-132. 